\title{
Estruturas de covariância de peso em função da idade de animais Nelore das regiões Sudeste e Centro-Oeste do Brasil
}

\author{
[Covariance structure of body weight in function of age for Nellore animals from Southeas \\ and Center West of Brazil]

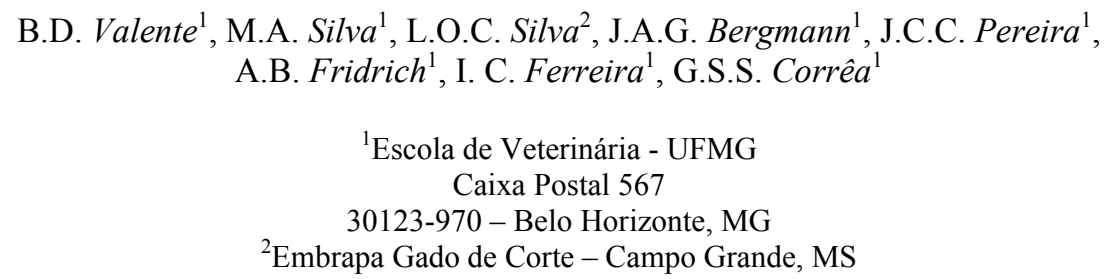

\section{RESUMO}

Dados de peso de animais da raça Nelore de 90 a 450 dias de idade das regiões Sudeste (SE) e Centro-Oeste (CO) do Brasil foram utilizados para comparar estruturas de (co)variância de efeitos aleatórios em função de idade estimadas para as duas regiões por meio de modelos de regressão aleatória. Componentes de (co)variância referentes aos coeficientes de regressão aleatória foram estimados por EMREML por meio do programa REMLF90. Os efeitos fixos de grupo contemporâneo e os efeitos aleatórios genético aditivo direto, genético aditivo materno e permanente de ambiente foram modelados por polinômios quadráticos de Legendre. As comparações envolveram estruturas de covariância e de correlação dos efeitos aleatórios, herdabilidades direta e materna e a razão entre variâncias genéticas de diferentes regiões. As herdabilidades e estruturas de covariância e de correlação apresentaram comportamento semelhante nas duas regiões. A variância residual e as variâncias de efeito permanente de ambiente foram menores no $\mathrm{CO}$, bem como a variância genética aditiva materna dos 150 aos 400 dias de idade. Trajetórias dos efeitos fixos em função de idade de diferentes grupos contemporâneos apresentaram diferentes formas, sugerindo a necessidade de estimar um conjunto de coeficientes de regressão específico para cada grupo contemporâneo. A variância do efeito genético aditivo materno apresentou maior heterogeneidade entre regiões do que a variância genética aditiva direta.

Palavras-chave: bovino de corte, função de covariância, regressão aleatória, trajetória de crescimento

\begin{abstract}
Body weight records from 90 to 450 days of age of Nellore animals from the Southeast and Center West of Brazil were used do estimate covariance structures of age dependent random effects for each region using random regression models. Covariance components of the regression coefficients were estimated by EMREML using the software REML90. The fixed effects of contemporary groups and additive genetic, additive maternal and permanent environment random effects were modeled by quadratic Legendre polynomials. The comparisons included structures of covariance and correlation of random effects, direct and maternal heritabitability and the ratio between genetic variances from different regions. The heritability, covariance and correlation structures showed similar patterns for both regions. Residual variance and permanent environment variances were smaller for Center West region as well as the maternal genetic additive from 150 to 400 days of age. Fixed effect trajectories in function of age of different contemporary groups showed different patterns, suggesting the necessity of specific set of regression coefficient estimates for each contemporary group. The maternal additive genetic variance showed higher heterogeneity between regions than the direct additive genetic variance.
\end{abstract}

Keywords: beef cattle, covariance function, growth trajectory, random regression

Recebido em 23 de março de 2007

Aceito em 31 de janeiro de 2008

E-mail: bvalente66@yahoo.com.br 


\section{INTRODUÇÃO}

A magnitude de ganhos genéticos de um programa de seleção depende de diversos fatores. Um deles é a eficiência na identificação dos animais com maior valor genético aditivo nas características a serem consideradas no critério de seleção (Falconer, 1989). Baixa disponibilidade de dados, má utilização dos dados disponíveis e a utilização de modelos estatísticos e metodologias de avaliação não adequadas levam à queda da acurácia de avaliação. A queda da eficiência de avaliação tem como conseqüência a predição de ganhos genéticos menos expressivos por parte do programa de seleção.

A característica peso é longitudinal e se modifica continuamente à medida que o animal fica mais velho. Considerando que o fenótipo de um animal é a soma de efeitos fixos e aleatórios, a magnitude desses efeitos bem como os parâmetros a eles atribuídos também se modificam à medida que o animal fica mais velho. $O$ peso corporal dos animais pode ser representado por um conjunto potencialmente infinito de pontos em diferentes idades, altamente correlacionados, que se modificam gradualmente até atingir um platô. $\mathrm{O}$ desafio é condensar essas informações de um animal em um conjunto manipulável de parâmetros com significado biológico (Arango et al., 2004).

Avaliações genéticas de peso em diferentes idades podem ser realizadas por intermédio de modelos multicaracterísticas (Marques et al., 2000). Esses modelos são utilizados pelos mais importantes programas de avaliação genética de animais Nelore, que avaliam os pesos ajustados em idades-padrão predefinidas. Ajustes do peso para animais que não foram avaliados exatamente na idade-padrão (a maioria) e eliminação de observações coletadas em idades distantes das consideradas padrão são procedimentos necessários, pois o modelo considera que as observações dos diferentes animais foram coletadas exatamente na mesma idade e estão submetidas aos mesmos parâmetros de (co)variância (Robbins et al., 2005). A utilização de dados ajustados, a eliminação de informações e a abordagem descontínua (em pontos) de uma característica que se modifica continuamente podem comprometer a acurácia de avaliação e o resultado da seleção. Nobre et al. (2003a) recomendaram a utilização de modelos multicaracterísticas com dados ajustados para idadespadrão. Porém, esse procedimento, de modo geral, não atribui o peso real que o animal teria à idadepadrão.
Modelos de regressão aleatória são mais adequados para a modelagem de pesos, pois consideram a modificação contínua do fenótipo e de todos os efeitos fixos e aleatórios que o compõem (bem como seus parâmetros) em função da idade do indivíduo. Dessa maneira, o peso é considerado como uma característica de dimensões infinitas (Kirkpatrick e Heckman, 1989). Modelos de regressão aleatória não necessitam de ajustes (Robbins et al., 2005), o que preserva a qualidade das informações e evita a eliminação de dados por serem coletados em idades distantes das definidas como padrão. Este modelo permite estimar estruturas de covariância em função da idade com relativamente poucos parâmetros (Gilmour, 2006). O melhor aproveitamento qualitativo e quantitativo das informações apresenta impacto positivo na acurácia das avaliações (Albuquerque, 2004; Bertrand et al., 2006).

Estudos a respeito da trajetória genética de crescimento de bovinos Nelore por meio de modelos de regressão aleatória já foram realizados (Albuquerque e Meyer, 2001; Nobre et al., 2003b), o que permitiu caracterizar as estruturas de covariância, correlação e herdabilidades em função da idade. Porém, não foi considerada a possibilidade de que as estruturas de variância podem ser diferentes em diferentes ambientes. Estruturas de covariância genética diferentes para cada ambiente exigem estratégias de seleção particulares para diferentes ambientes, pois critérios de seleção estabelecidos com valores genéticos preditos com base em estruturas genéticas não adequadas poderiam resultar em ganhos genéticos diferenciados para cada ambiente do que seria esperado. Diferenças na estrutura de covariância entre coeficientes de regressão aleatória levariam também a diferentes resultados, caso os coeficientes de regressão aleatória fossem utilizados como critério de seleção. Dessa maneira, há necessidade de se caracterizarem estruturas de covariância para diferentes ambientes.

O objetivo do presente estudo foi comparar as estruturas de (co)variância estimadas para dados das regiões Centro-Oeste e Sudeste, que são as duas regiões mais importantes na criação de bovinos da raça Nelore no Brasil.

\section{MATERIAL E MÉTODOS}

As informações utilizadas neste estudo são provenientes do Controle de Desenvolvimento Ponderal da Associação Brasileira de Criadores de Zebu e cedidas pela Embrapa - Gado de Corte, 
localizada em Campo Grande, MS. O conjunto de dados utilizado constitui pesagens registradas de 926.391 animais da raça Nelore entre 90 e 450 dias de idade, filhos de 15.721 touros e 376.509 vacas. A distribuição das informações, ao longo do intervalo de idade considerado é representada na Fig. 1. Os dados foram coletados entre 1975 e 2003.

Para comparar as estruturas de covariância estimadas nas regiões Centro-Oeste e Sudeste, foi feita a classificação dos dados em cinco grandes regiões do Brasil (Norte, Nordeste, Centro-Oeste, Sudeste e Sul) e a eliminação das observações provenientes das regiões Norte, Nordeste e Sul. Posteriormente, os animais foram divididos em grupos contemporâneos, definidos por sexo, ano de nascimento, propriedade e estação de nascimento (janeiro a março, abril a junho, julho a setembro, outubro a dezembro). Foram eliminadas observações de animais que, na data do nascimento, tinham pais ou mães com menos de 450 dias de idade ou mães com mais de 20 anos de idade, animais com menos de três pesos registrados e animais sem registro de pai ou mãe, ou sem registro de propriedade. Apenas observações dos que foram criados em pasto foram consideradas. Após divisão das idades à pesagem em 12 classes de 30 dias, foram eliminadas as observações que apresentavam valores maiores ou menores do que três unidades de desvio-padrão de diferença em relação à média da classe.

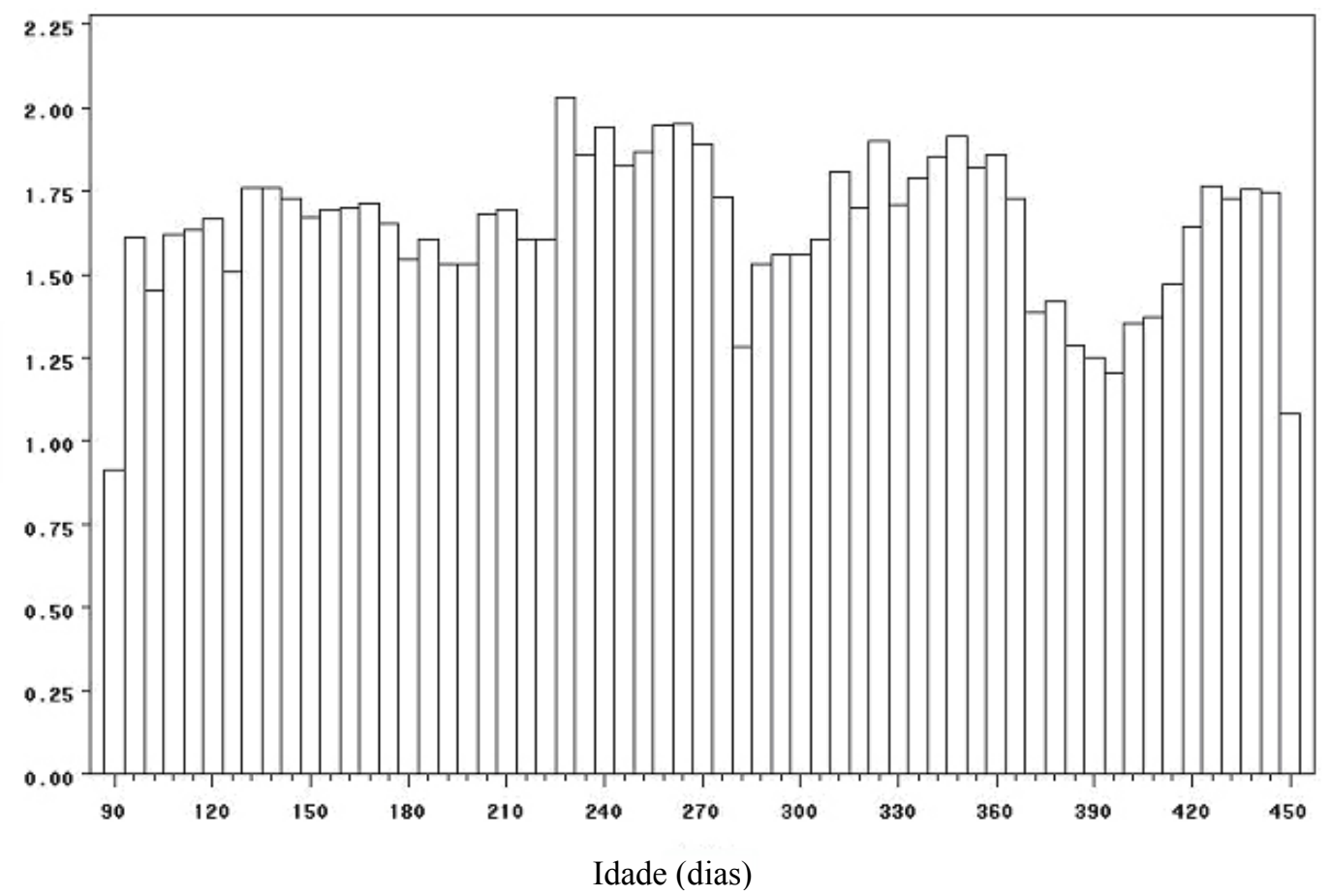

Figura 1. Distribuição (\%) dos pesos dos animais segundo o intervalo de idade (90 a 450 dias).

Para garantir estimativa acurada da trajetória de crescimento fixa atribuída a cada grupo contemporâneo, apenas os grupos com maior número de informações foram considerados na análise. Para isso, foram eliminados os grupos contemporâneos com menos de 200 animais. O efeito fixo da idade da mãe ao parto foi representado por covariáveis lineares e quadráticas relativas à sua idade.
Três arquivos de dados foram definidos após a edição das informações: o primeiro arquivo com todas as observações coletadas na região Sudeste (SE), o segundo arquivo com todas as observações coletadas na região Centro-Oeste (CO) e o terceiro arquivo com observações das duas regiões (T). A descrição dos três arquivos encontra-se na Tab. 1. 
Tabela 1. Descrição dos arquivos contendo pesos dos animais coletados nas regiões Sudeste (SE), CentroOeste (CO) e o arquivo com todos os pesos (T)

\begin{tabular}{lccc}
\hline & SE & CO & T \\
\hline Observações & 49742 & 32838 & 82580 \\
Animais com observações & 13863 & 9424 & 23287 \\
Animais incluídos na matriz de parentesco & 24750 & 16092 & 40741 \\
Grupos contemporâneos & 49 & 36 & 85 \\
Propriedades & 14 & 9 & 23 \\
Reprodutores & 577 & 432 & 917 \\
Matrizes & 10574 & 6691 & 17256 \\
\hline
\end{tabular}

O modelo de regressão aleatória utilizado teve como efeitos fixos os grupos contemporâneos e como efeitos linear e quadrático a idade da mãe ao parto. A cada grupo contemporâneo foi atribuída uma trajetória fixa de crescimento entre as idades de 90 a 450 dias, partindo da pressuposição de que diferentes grupos contemporâneos podem apresentar diferentes formas de trajetória de crescimento. Da mesma maneira, o efeito fixo da idade da mãe ao parto foi estimado para cada grupo contemporâneo.

Todos os efeitos considerados como função da idade do animal que produz a observação foram representados por coeficientes de regressão constante, linear e quadrático sobre os respectivos polinômios de Legendre. A escolha feita pelo modelo quadrático para descrever as variáveis aleatórias baseou-se no fato de que análises semelhantes, como a realizada por Nobre et al. (2003b), demonstraram boa representação dos efeitos por polinômios cúbicos, sendo que as matrizes de covariâncias entre coeficientes de regressão aleatória dos diferentes efeitos aleatórios apresentaram sistematicamente valores muito baixos em um dos autovalores, e o intervalo de idade avaliado pelos autores era o dobro do utilizado no presente estudo. A operacionalidade computacional também foi considerada na escolha do modelo, uma vez que a utilização de maiores ordens polinomiais apresenta, como conseqüência, o aumento dos custos computacionais da análise.

O modelo utilizado pode ser definido como:

$$
\mathrm{y}_{\text {fghij }}=\sum_{\mathrm{k}=0}^{2} \mathrm{gc}_{\mathrm{fk}} \phi_{\mathrm{k}}(\mathrm{i})+\sum_{\mathrm{n}=1}^{2} \mathrm{~b}_{\mathrm{fn}} \mathrm{m}_{\mathrm{n}}(\mathrm{g})+\sum_{\mathrm{k}=0}^{2} \mathrm{~d}_{\mathrm{hk}} \phi_{\mathrm{k}}(\mathrm{i})+\sum_{\mathrm{k}=0}^{2} \mathrm{mat}_{\mathrm{jk}} \phi_{\mathrm{k}}(\mathrm{i})+\sum_{\mathrm{k}=0}^{2} \mathrm{p}_{\mathrm{hk}} \phi_{\mathrm{k}}(\mathrm{i})+\mathrm{e}_{\mathrm{fghij}},
$$

em que $y_{f g h i j}$ é a observação $i$ do animal $h$ no grupo contemporâneo $f$, da mãe $j$ com idade $g$; $g c_{f k}$ é o coeficiente de regressão fixo $k$ atribuído ao grupo contemporâneo $f ; b_{f n}$ é o coeficiente de regressão fixo $n$ de efeito da idade da mãe ao parto no grupo contemporâneo $f ; d_{h k}$ e $p_{h k}$ são os coeficientes de regressão aleatória $k$ de efeito genético aditivo direto e permanente de ambiente atribuídos ao animal $h$; mat $_{j k}$ é o coeficiente de regressão aleatória $k$ do efeito genético aditivo materno atribuído à mãe $j ; \phi_{k}(i)$ é o polinômio de Legendre $k$ normalizado atribuído à idade transformada (escala de -1 a 1) na coleta da observação $i ; m_{n}(g)$ é o polinômio "natural" $n$ atribuído à idade da mãe ao parto $g$; e $e_{f g h i j}$ é o efeito residual atribuído a cada observação $y_{f g h i j}$. No modelo apresentado, a cada grupo contemporâneo são atribuídos efeitos fixos da idade sobre o peso do animal, representados por coeficientes de regressão relativos aos polinômios de Legendre considerados, além de covariáveis lineares e quadráticas em função da idade da mãe ao parto.

Em notação matricial, o modelo pode ser representado da seguinte forma:

$y=X_{1} c+X_{2} b+Z_{1} d+Z_{2} m+Z_{3} p+e$,

em que $y$ é o vetor com as observações; $c$ é o vetor com os coeficientes de regressões relativos aos efeitos fixos de grupos contemporâneos; $b$ é o vetor com as covariáveis relativas à idade da mãe ao parto; $d, m$ e $p$ são vetores com coeficientes de regressão aleatória relativos aos efeitos genético aditivo direto, genético aditivo materno e permanente de ambiente, respectivamente; $X_{1}, X_{2}, Z_{1}, Z_{2}, Z_{3}$ são matrizes de incidência nas observações dos efeitos nos vetores $c, b, d, m$ e $p$, respectivamente, contendo os polinômios de Legendre relativos a cada grau e idade (à exceção de $X_{2}$, que contém polinômios naturais relativos aos efeitos lineares $\mathrm{e}$ 
quadráticos de idade da mãe ao parto); e $e$ é o vetor dos resíduos.

Adicionalmente foi considerado que

$$
\mathrm{V}\left[\begin{array}{c}
\mathrm{d} \\
\mathrm{m} \\
\mathrm{p} \\
\mathrm{e}
\end{array}\right]=\left[\begin{array}{cccc}
\mathrm{A} \otimes \mathrm{G}_{\mathrm{d}} & \mathrm{A} \otimes \mathrm{G}_{\mathrm{dm}} & 0 & 0 \\
\mathrm{~A} \otimes \mathrm{G}_{\mathrm{dm}}^{\prime} & \mathrm{A} \otimes \mathrm{G}_{\mathrm{m}} & 0 & 0 \\
0 & 0 & \mathrm{I}_{\mathrm{h}} \otimes \mathrm{P} & 0 \\
0 & 0 & 0 & \mathrm{I}_{\mathrm{n}} \sigma_{\mathrm{e}}^{2}
\end{array}\right]
$$

em que $G_{d}, G_{m}$ e $P$ são as matrizes $3 \times 3$ de covariância entre os coeficientes de regressão aleatória que modelam os efeitos genético aditivo direto, genético aditivo materno e permanente de ambiente; $\sigma_{\mathrm{e}}^{2}$ é a variância residual; $G_{d m}$ é a matriz de covariância entre os coeficientes de regressão atribuídos aos efeitos genético aditivo direto e materno; $A$ é a matriz de numeradores do coeficiente de parentesco de Wright; $h$ é o número de animais que possuem observações; e $n$ é o número total de observações. Desta maneira, a covariância entre os efeitos genético aditivo direto e materno foi considerada na análise.

O modelo foi utilizado para estimação de componentes de (co)variância, efeitos fixos e predição de efeitos aleatórios nos três arquivos anteriormente definidos. Os elementos das matrizes $G_{d}, G_{m}, G_{d m}$ e $P$, bem como $\sigma_{e}^{2}$, foram estimados por meio do programa REMLF90 (Misztal, 2001) que utiliza a maximização da esperança da função de máxima verossimilhança restrita (EMREML) com aceleração de convergência. $\mathrm{O}$ critério de convergência utilizado, definido pelo quadrado médio das diferenças entre soluções consecutivas, foi de $1 \times 10^{-11}$.

A divisão regional das observações possibilita avaliações distintas, o que permite comparar as estruturas de covariância e de correlação dos efeitos aleatórios, além das herdabilidades direta e materna das duas regiões. A heterogeneidade de variâncias foi estudada para efeitos genéticos aditivos direto e materno, pois sua existência indicaria interação genótipo x região.

\section{RESULTADOS E DISCUSSÃO}

Efeitos fixos da idade sobre o peso dos animais em uma amostra de seis grupos contemporâneos considerados na análise dos dados referentes a todos os animais (T) estão representados na Fig. 2. As curvas que representam os efeitos de diferentes grupos contemporâneos apresentam formas variadas, o que indica que é apropriado estimar para cada grupo contemporâneo um conjunto de coeficientes de regressão, partindo da pressuposição de que pode existir interação entre níveis de efeito fixo e forma da trajetória de crescimento. Albuquerque e Meyer (2001), ao avaliarem trajetórias de crescimento de bovinos de corte da raça Nelore do nascimento aos 630 dias de idade, atribuíram a cada grupo contemporâneo um efeito fixo simples, que é somado à curva que modela a trajetória média de crescimento da população, definida por um conjunto único de coeficientes de regressão. Essa estratégia de estimar os efeitos fixos em função da idade considera que as trajetórias de crescimento fixas atribuídas a diferentes grupos contemporâneos podem apresentar diferentes alturas na representação gráfica, mas não diferentes inclinações ou diferentes curvaturas na trajetória de crescimento.

Os componentes de (co)variância atribuídos aos coeficientes de regressão que representam cada efeito aleatório do peso em função da idade, estimados com critério de convergência definido por quadrado médio das diferença entre soluções consecutivas menores que $1 \times 10^{-11}$, são apresentados na Tab. 2. Como observado por Albuquerque e Meyer (2001), o coeficiente de regressão aleatória relativo ao intercepto é o que apresenta maior variância em todos os efeitos aleatórios estruturados. As autoras ainda relatam que as correlações entre os coeficientes de regressão relativos ao intercepto e ao efeito linear foram positivas para todos os efeitos aleatórios, com valores moderados a altos. No presente estudo, as correlações entre esses dois coeficientes de regressão nos diferentes efeitos aleatórios foram sempre positivas, mas altos valores só foram estimados para o efeito de ambiente permanente (acima de 0,996). Já as correlações entre interceptos e coeficientes quadráticos foram sempre negativas e, conforme relatado por Albuquerque e Meyer (2001), são valores que variam de próximos a zero a próximos da unidade. A tendência citada pelas autoras de obtenção de estimativas de correlações entre esses coeficientes mais negativas para o efeito genético aditivo materno em relação ao efeito genético aditivo direto se 
confirmou nas três análises realizadas. As correlações entre os coeficientes de regressão linear e quadrático foram positivas, exceto para o efeito permanente de ambiente.

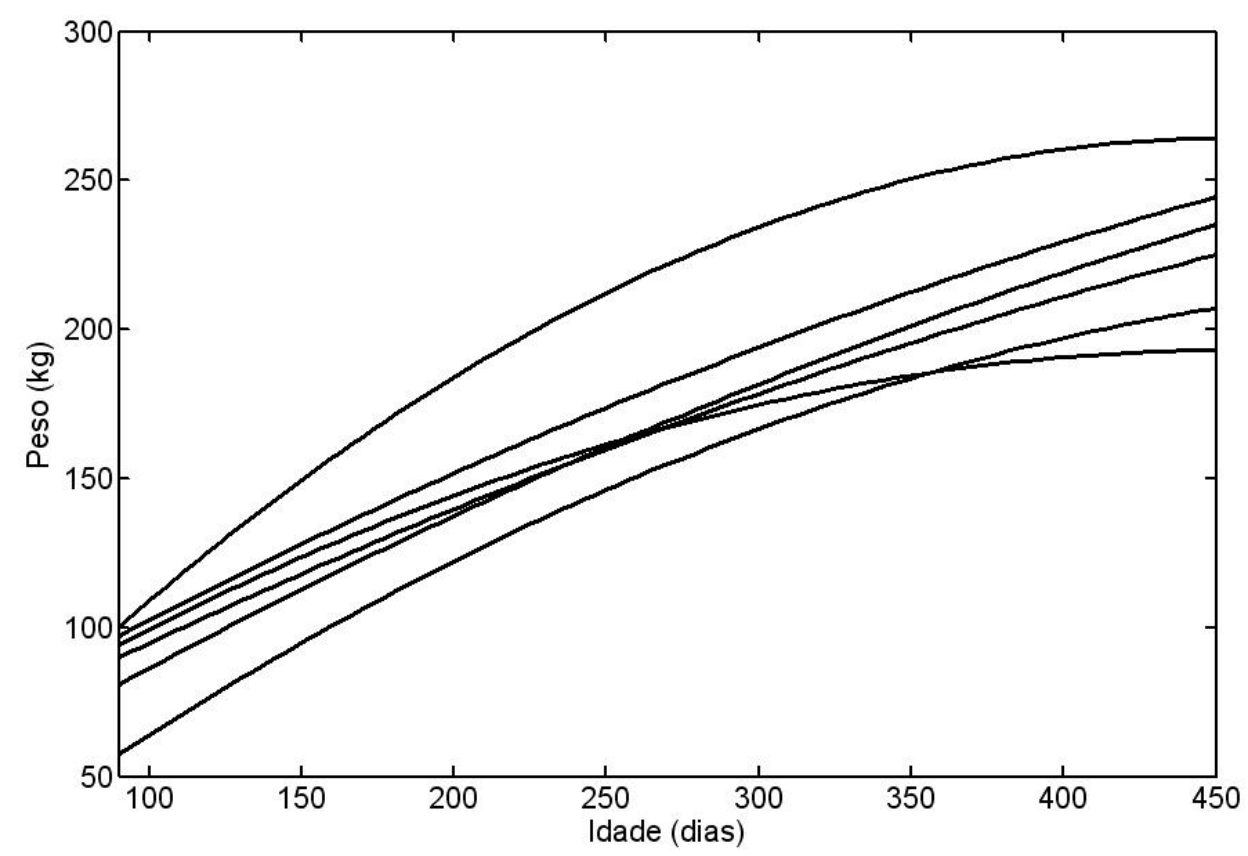

Figura 2. Efeito da idade (dias), considerada como efeito fixo, sobre o peso dos animais em cada um de seis grupos contemporâneos amostrados em todas as regiões estudadas.

Os autovalores atribuídos às matrizes de covariância dos coeficientes de regressão dos efeitos aleatórios são apresentados na Tab. 2. A existência de pelo menos um autovalor muito pequeno em cada efeito aleatório (menor que $0,29 \%$ em relação ao maior autovalor) indica que a equação quadrática proporcionou ajuste suficiente de todos os efeitos nas três análises (Nobre et al., 2003b).

As variâncias estimadas dos efeitos aleatórios em função da idade estão representadas na Fig. 3. Todos os efeitos aleatórios apresentaram heterogeneidade de variância ao longo do intervalo de idade considerado, com comportamento semelhante para SE, CO e T. A variância atribuída ao efeito genético aditivo direto foi a que apresentou menor heterogeneidade. Nas análises relativas a T e SE, a variância genética aditiva direta apresentou leve queda no início do intervalo, e voltou a crescer, aproximadamente, a partir dos 200 dias de idade. Essa depressão na estrutura de variância não foi observada em estudos de outros autores, nessa idade. Uma possível explicação para esse comportamento da variância é que tal modelo de avaliação pode apresentar artefatos (problemas intrínsecos do modelo de regressão aleatória) nas estimativas de variância nos extremos do intervalo de idade considerado (Arango et al., 2004; Gilmour, 2006), o que pode estar causando superestimação de variância no extremo inicial. A variância genética aditiva direta atribuída a $\mathrm{T}$ apresentou, ao final do intervalo, crescimento menos acelerado do que o apresentado na análise de SE e CO. Porém, nas três análises, a variância do efeito apresentou clara tendência de crescimento ao final do intervalo, o que correspondeu aos resultados obtidos por Albuquerque e Meyer (2001) e Nobre et al. (2003b), ao avaliarem trajetória de crescimento de bovinos da raça Nelore do nascimento aos 683 dias, e por Legarra et al. (2004), ao avaliarem trajetória de crescimento de bovinos de corte da raça Gelbvieh. 
Tabela 2. Componentes de (co)variância (diagonal e acima da diagonal), correlações (abaixo da diagonal) atribuídas aos coeficientes de regressão do intercepto $\left(b_{0}\right)$, linear $\left(b_{1}\right)$ e quadrático $\left(b_{2}\right)$, variâncias residuais e autovalores das matrizes de covariância do efeito genético aditivo direto, genético aditivo materno e do efeito permanente de ambiente para peso, estimados para todos os dados (T) e para as regiões Sudeste (SE) e CentroOeste $(\mathrm{CO})$

\begin{tabular}{lccccccccc}
\hline \multicolumn{10}{c}{$\mathrm{c}$} \\
\hline & \multicolumn{10}{c}{ Genético aditivo direto } & \multicolumn{2}{c}{ Genético aditivo materno } & \multicolumn{2}{c}{ Permanente de ambiente } \\
\cline { 2 - 11 } & $b_{0}$ & $b_{1}$ & $b_{2}$ & $b_{0}$ & $b_{1}$ & $b_{2}$ & $b_{0}$ & $b_{1}$ & $b_{2}$ \\
\hline$b_{0}$ & 204,2 & 6,154 & $-1,661$ & 157,6 & 5,572 & $-58,85$ & 409,1 & 172,5 & $-0,4535$ \\
$b_{1}$ & 0,1230 & 12,26 & 0,5084 & 0,1307 & 11,53 & 5,318 & 0,9966 & 73,24 & $-0,1838$ \\
$b_{2}$ & $-0,5506$ & 0,6880 & 0,0445 & $-0,8970$ & 0,2996 & 27,32 & $-0,4730$ & $-0,4531$ & 0,0022 \\
\hline Autovalores & 204,41 & 120,890 & 0,0052 & 180,31 & 15,817 & 0,32218 & 481,91 & 0,4280 & 0,0016 \\
\cline { 2 - 9 }
\end{tabular}

\begin{tabular}{|c|c|c|c|c|c|c|c|c|c|}
\hline Residual & \multicolumn{9}{|c|}{215,6} \\
\hline \multicolumn{10}{|c|}{ SE } \\
\hline & \multicolumn{3}{|c|}{ Genético aditivo direto } & \multicolumn{3}{|c|}{ Genético aditivo materno } & \multicolumn{3}{|c|}{ Permanente de ambiente } \\
\hline & $b_{0}$ & $b_{1}$ & $b_{2}$ & $b_{0}$ & $b_{1}$ & $b_{2}$ & $b_{0}$ & $b_{1}$ & $b_{2}$ \\
\hline$b_{0}$ & 216,6 & 10,54 & $-1,884$ & 155,8 & 8,104 & $-63,61$ & 457,2 & 184,7 & $-1,338$ \\
\hline$b_{1}$ & 0,1656 & 18,68 & 1,124 & 0,1713 & 14,36 & 3,747 & 0,9963 & 75,2 & $-0,5279$ \\
\hline$b_{2}$ & $-0,3938$ & 0,7999 & 0,1056 & $-0,9254$ & 0,1796 & 30,32 & $-0,8212$ & $-0,7989$ & 0,0058 \\
\hline Autovalores & 217,18 & 18,203 & 0,0078 & 182,62 & 17,325 & 0,5338 & 531,9 & 0,50293 & 0,0016 \\
\hline
\end{tabular}

Residual

231,8

\begin{tabular}{|c|c|c|c|c|c|c|c|c|c|}
\hline \multicolumn{10}{|c|}{$\mathrm{CO}$} \\
\hline & \multicolumn{3}{|c|}{ Genético aditivo direto } & \multicolumn{3}{|c|}{ Genético aditivo materno } & \multicolumn{3}{|c|}{ Permanente de ambiente } \\
\hline & $b_{0}$ & $b_{I}$ & $b_{2}$ & $b_{0}$ & $b_{I}$ & $b_{2}$ & $b_{0}$ & $b_{I}$ & $b_{2}$ \\
\hline$b_{0}$ & 220,2 & 13,82 & $-1,645$ & 126,3 & 3,262 & $-43,81$ & 323,2 & 136,8 & $-0,2227$ \\
\hline$b_{1}$ & 0,2609 & 12,75 & 0,2902 & 0,0837 & 12,03 & 8,097 & 0,9967 & 58,31 & $-0,0887$ \\
\hline$b_{2}$ & $-0,6198$ & 0,4544 & 0,0319 & $-0,8159$ & 0,4885 & 22,84 & $-0,2865$ & $-0,2688$ & 0,0018 \\
\hline Autovalores & 221,13 & 11,847 & 0,0066 & 142,36 & 18,516 & 0,2943 & 381,17 & 0,3452 & 0,0016 \\
\hline
\end{tabular}

A variância estimada para o efeito genético aditivo materno foi muito baixa aos 90 dias de idade e, logo em seguida, apresentou acentuado crescimento até o intervalo de 200 a 250 dias, quando o crescimento diminuiu de intensidade até atingir o pico, aproximadamente aos 250 dias. Após essa idade, a variância do efeito genético aditivo materno mostrou pronunciada queda. $\mathrm{O}$ crescimento dos valores de variância do efeito genético aditivo materno relativos a $\mathrm{CO}$ não apresentou a mesma intensidade de crescimento observada na análise de T e SE. O efeito genético aditivo materno resultou, nas três análises realizadas, em pequeno aumento da variância, sem nenhuma explicação biológica após, aproximadamente, 400 dias de idade, provavelmente causado por artefatos do modelo de regressão aleatória. A estimativa da variância no início do intervalo (logo após 90 dias de idade) foi próxima de zero, o que parece não condizer com a realidade do efeito nessa idade. O comportamento da variância do efeito genético aditivo materno foi semelhante ao observado por Albuquerque e Meyer (2001), inclusive no que diz respeito ao pequeno crescimento da variância no final do intervalo. Porém, valores máximos da variância, observados pelas autoras, foram atingidos aproximadamente aos 200 dias de idade. Nobre et al. (2003b) e Legarra et al. (2004), ao modelarem a variância do efeito genético aditivo materno, não observaram decréscimo significativo após o período de desmama. Os valores de variância obtidos no presente estudo foram maiores do que os apresentados por Albuquerque e Meyer (2001) e Nobre et al. (2003b). Uma possível explicação para isso é o fato de que, no presente estudo, não foi considerado o efeito de ambiente permanente materno, que pode ter se confundido, em parte, com o efeito genético aditivo materno. Segundo Meyer (1992), há evidências de que grande parte da 
variância do efeito materno é considerada pela inclusão de apenas um efeito materno. Partição da variância nos efeitos genéticos aditivos materno e

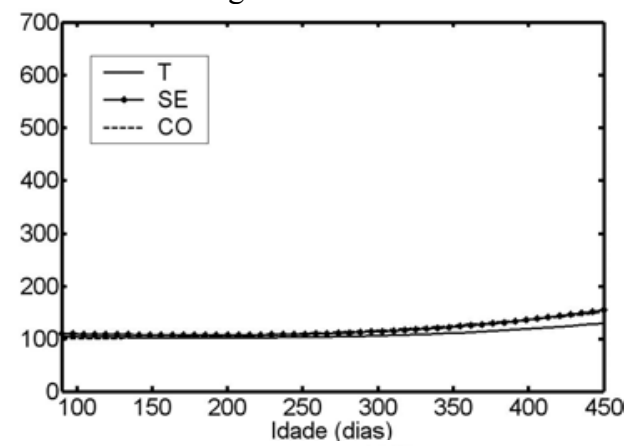

permanente de ambiente materno em dados de campo apresenta dificuldades, mesmo em análises simples univariadas.

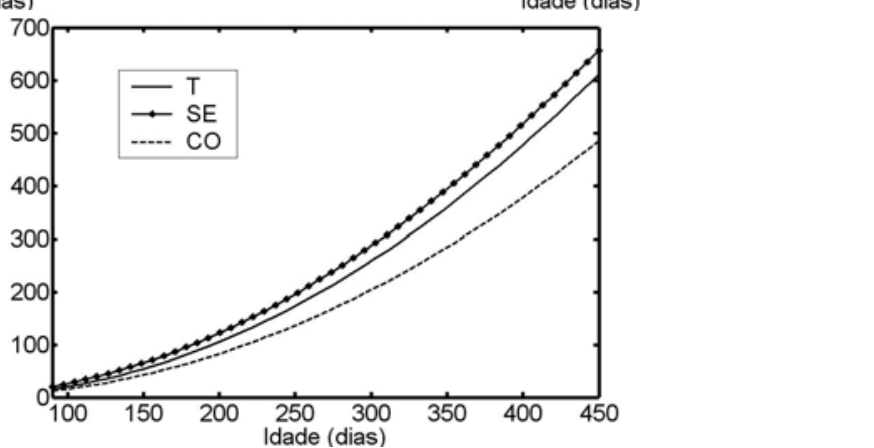

Figura 3. Variâncias dos efeitos genético aditivo direto, genético aditivo materno e permanente de ambiente (superior esquerdo, superior direito e inferior, respectivamente) do peso dos animais em função da idade estimadas para ambas as regiões (T), e para as regiões Sudeste (SE) e Centro-Oeste (CO).

A variância do efeito permanente de ambiente foi baixa aos 90 dias de idade, mas posteriormente apresentou crescimento contínuo e acelerado até os 450 dias de idade. Esse padrão foi comum para todas as três análises, mas com crescimento pouco menor para o CO. Aumento constante da variância do efeito permanente de ambiente também foi relatado por Albuquerque e Meyer (2001) e Nobre et al. (2003b), em bovinos Nelore, por Sakaguti (2000), na avaliação da trajetória de crescimento de bovinos Tabapuã, e por Cue et al. (2006), na avaliação da trajetória de crescimento de novilhas de leite em Quebec, Canadá. Esse comportamento ao longo do intervalo de idade ocorreu, provavelmente, em razão do caráter cumulativo desse efeito (Schaffer, 2001), o que causou intensa heterogeneidade de variância.

As estruturas de covariância estimadas para cada efeito aleatório e a covariância fenotípica estão representadas na Fig. 4. As estruturas atribuídas aos efeitos aleatórios estimados para SE e CO, por apresentarem o mesmo padrão de comportamento em função da combinação de idades mostrado pelas estruturas estimadas para $\mathrm{T}$, não serão apresentadas. As superfícies que representam as covariâncias dos efeitos genéticos aditivos diretos para $\mathrm{T}$ e $\mathrm{SE}$ apresentaram leve depressão na covariância dos 90 aos 200 dias. Após essa faixa de idade, as covariâncias estimadas nas três análises apresentaram inclinação em direção ao aumento de idade nos eixos x e y, padrão observado também por Sakaguti (2000) em bovinos de corte Tabapuã, e por Arango et al. (2004), na avaliação da trajetória de crescimento de matrizes de diferentes raças e cruzamentos de corte. A superfície que representa a estrutura de covariância do efeito permanente de ambiente também apresentou inclinação em direção ao aumento de idade nos eixos $\mathrm{x}$ e $\mathrm{y}$, porém de modo mais intenso que a observada no efeito genético aditivo direto. A superfície que representa a covariância fenotípica também mostrou valores crescentes em direção ao aumento da idade. Após os 250 dias de idade, a inclinação se tornou menos intensa em razão da diminuição dos valores de covariância do efeito genético aditivo materno, mas a inclinação 
voltou a se tornar mais intensa após, aproximadamente, 350 dias de idade, impulsionada principalmente pelo crescimento acelerado dos valores de covariância do efeito permanente de ambiente. A crista observada na diagonal da superfície é atribuída à variância do resíduo, que não foi estruturada no modelo, sendo, por esse motivo, constante para todas as idades do intervalo e apresentando covariância nula para medidas em diferentes idades. A variância fenotípica estimada para $\mathrm{CO}$ foi menor que a estimada para $\mathrm{SE}$, em razão dos menores valores de variância do efeito genético aditivo materno, ambiente permanente e residual.

Parte dos efeitos da interação genótipo $\mathrm{x}$ ambiente ocorre em razão da heterogeneidade de variância genética em diferentes ambientes, o que causa queda da eficiência, por exemplo, na predição do desempenho de filhos de reprodutores em ambientes diferentes daqueles em que esses reprodutores foram avaliados. A razão entre as variâncias do efeito genético aditivo direto de $\mathrm{CO}$ e de $\mathrm{SE}$ em função da idade, apresentadas na Fig. 5, mostrou valores próximos de 1 em todo o intervalo de idade, o que indica inexpressiva heterogeneidade de variâncias entre as duas regiões para esse efeito. Portanto, não ocorreram prejuízos na predição de desempenho e na comparação de reprodutores com informações em diferentes regiões. Por sua vez, o efeito genético aditivo materno mostrou maior heterogeneidade de variância entre regiões. No intervalo de idades em que a variância do efeito genético materno teve os maiores valores, a variância estimada para $\mathrm{CO}$ correspondeu a menos de $80 \%$ da variância desse efeito para SE. No intervalo de maior heterogeneidade (entre 300 e 350 dias), a razão entre variâncias atingiu o valor de 0,736. Dessa maneira, a comparação entre efeitos genéticos maternos preditos para animais avaliados em diferentes regiões estaria comprometida. Nos dois extremos de idade, houve inversão brusca da proporção, mas a susceptibilidade da estrutura de variância estimada a artefatos nas idades próximas a 90 e 450 dias levou à forte suspeita de que essa inversão de proporções não correspondeu à realidade.
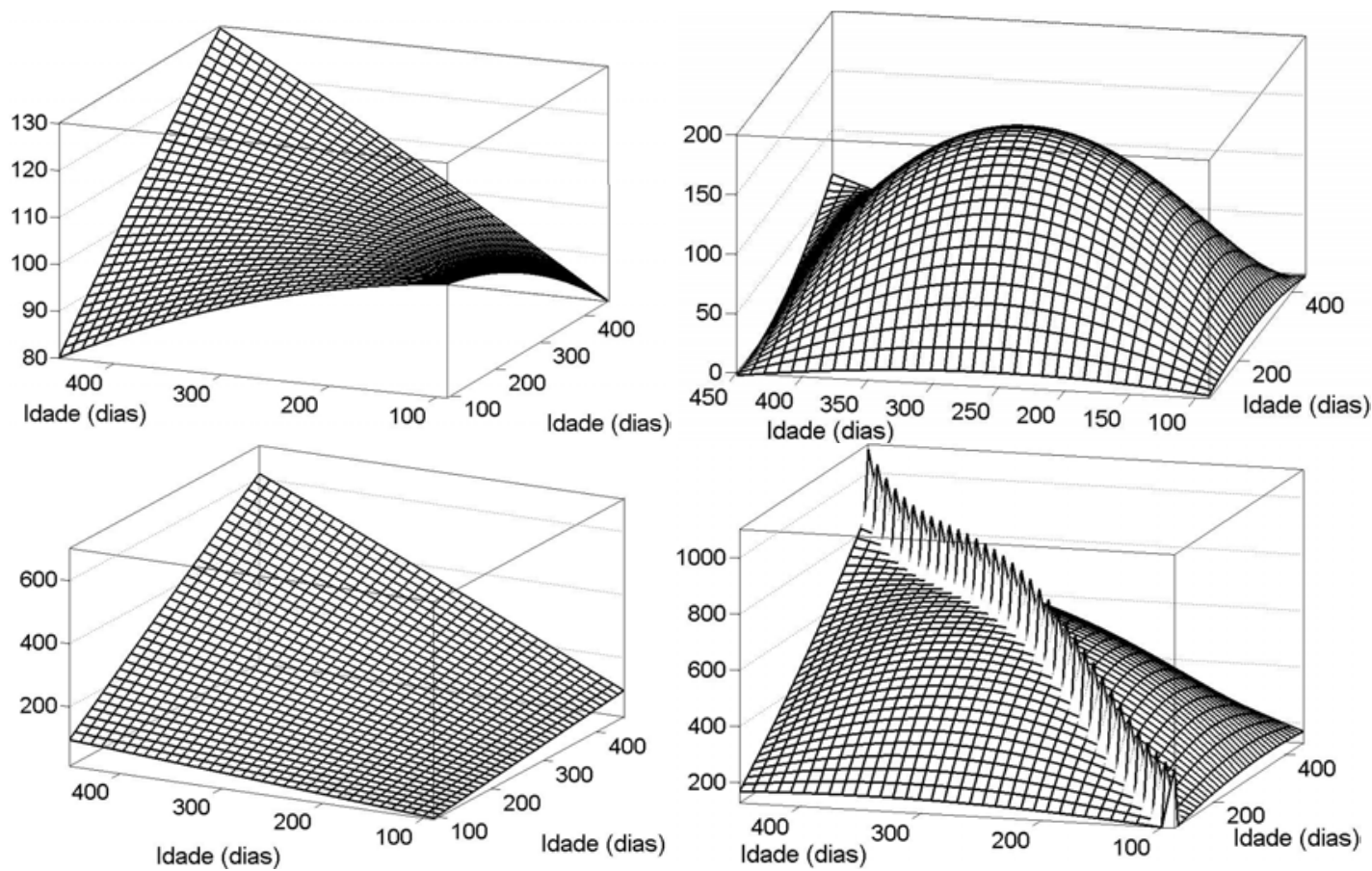

Figura 4. Estruturas de covariância dos efeitos genético aditivo direto (superior esquerdo), genético aditivo materno (superior direito), permanente de ambiente (inferior esquerdo) e estrutura de covariância fenotípica (inferior direito) do peso em função da idade estimadas para dados de ambas as regiões $(\mathrm{T})$. 


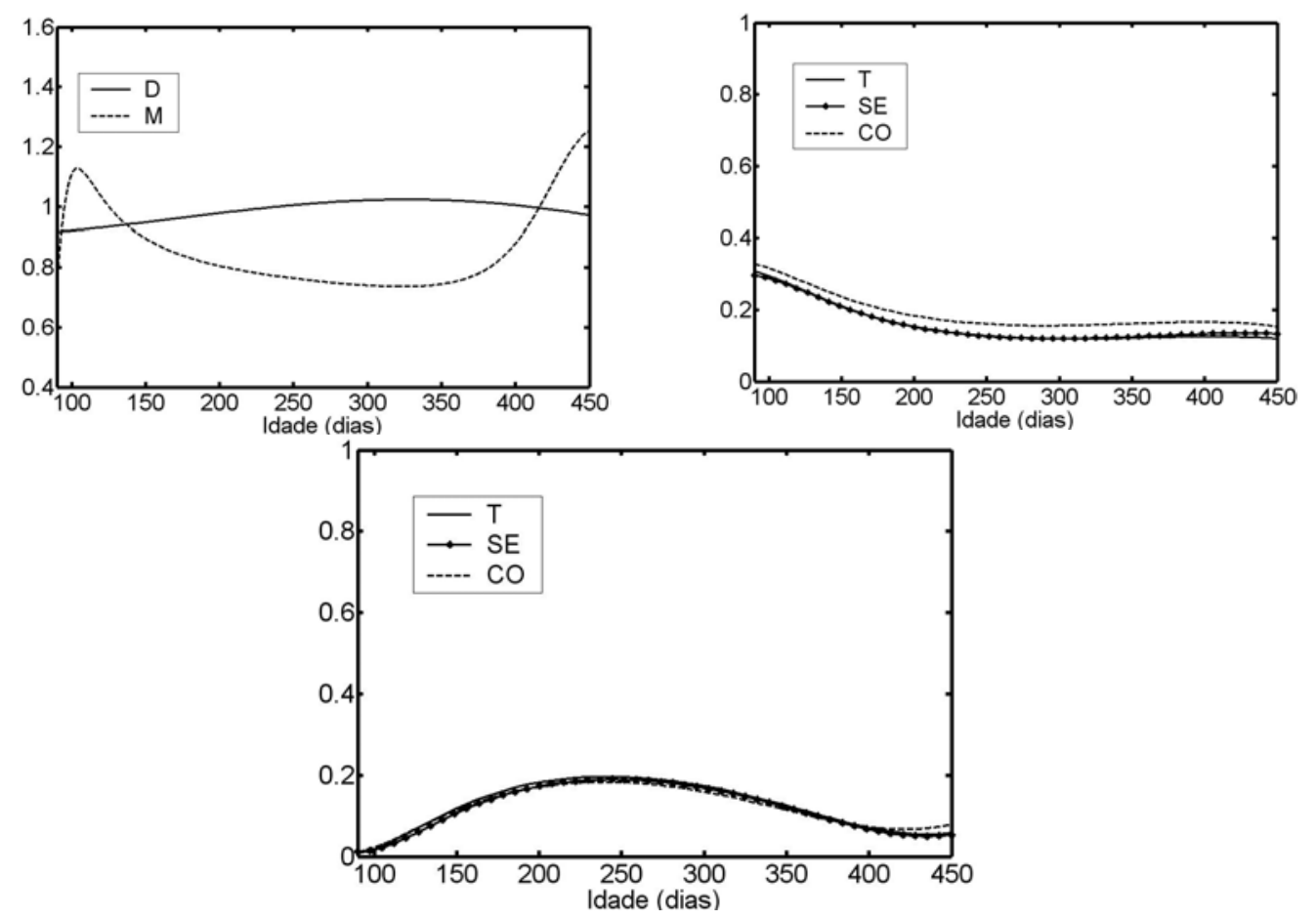

Figura 5. Razão entre variâncias de peso em função da idade nas regiões Centro-Oeste (CO) e Sudeste (SE) dos efeitos genético aditivo direto (D) e genético aditivo materno (M) (superior esquerdo), herdabilidades direta (superior direito) e materna (inferior) do peso em função da idade do animal, estimadas para ambas as regiões (T), e para as regiões Sudeste (SE) e Centro-Oeste (CO).

Os maiores valores de herdabilidade direta (Fig. 5) foram estimados para peso aos 90 dias de idade, entre 0,30 e 0,33. Em seguida, houve queda até se estabilizarem, após os 250 dias de idade, próximos a 0,12 para T e SE e 0,16 para CO. Maiores valores de herdabilidade eram esperados para dados da região Centro-Oeste, uma vez que as variâncias residuais de ambiente permanente e genética aditiva materna para essa região são menores do que as de $\mathrm{T}$ e $\mathrm{SE}$, enquanto a variância genética aditiva direta mostrou-se bem próxima às estimadas em $\mathrm{T}$ e SE. Albuquerque e Meyer (2001) também obtiveram maiores estimativas de herdabilidade para as menores idades $(0,32)$, com queda para 0,14 em idades posteriores. Comportamento semelhante da herdabilidade foi observado por Cue et al. (2006). Os valores de herdabilidade após a estabilização foram coerentes com os relatados por Eler et al. (1995), Albuquerque e Meyer (2001), Nobre et al. (2003b) e Cue et al. (2006), que observaram valores menores que 0,20. Albuquerque e Meyer (2001) observaram aumento significativo nos valores de herdabilidade na metade final do intervalo de idade. As herdabilidades estimadas no presente estudo não mostraram esse aumento. Os autores citados consideraram idades mais altas nas quais houve aumento da variância genética aditiva direta e queda dos valores de variância genética aditiva materna.

As herdabilidades maternas estimadas (Fig. 5) foram menores nos extremos e maiores durante a fase que coincide com os maiores valores de variância genética aditiva materna, superando as herdabilidades diretas nesse período. Os valores da herdabilidade materna estimados nas três análises foram idênticos para todo o intervalo de idades considerado, destacando-se os resultados obtidos para $\mathrm{CO}$, em que a menor variância do efeito genético aditivo materno parece ter sido compensada pelos menores valores de variância residual e do efeito permanente de ambiente. Comportamentos semelhantes foram observados por Albuquerque e Meyer (2001) e Nobre et al. (2003b). As primeiras autoras salientaram que a herdabilidade direta tende a ser menor nas idades em que a herdabilidade materna apresenta maiores valores. Dessa forma, a seleção com 
base na habilidade materna poderia resultar em maiores ganhos no desempenho nessa faixa de idade do que os ganhos estimados para a seleção de animais com maiores valores genéticos aditivos diretos. Essa estratégia de seleção, porém, poderia resultar em matrizes que produzem mais leite e, conseqüentemente, apresentam maior demanda nutricional.

As estruturas de correlação em função da combinação de idades estimadas para cada efeito aleatório de $\mathrm{T}$ são apresentadas na Fig. 6. A exemplo das estruturas de covariância, as correlações estimadas para SE e CO mostraram o mesmo comportamento das estimadas para $\mathrm{T}$ e não serão apresentadas. As correlações genéticas aditivas diretas apresentaram-se em uma superfície dominada por altos valores, à exceção das correlações estimadas entre os extremos das idades estudadas. Quanto maior o intervalo entre dois pontos, menor é a correlação estimada, porém sempre maior que 0,69. Resultados semelhantes foram observados por Sakaguti (2000) que estimou, por meio de modelo multicaracterística, correlações genéticas aditivas diretas sempre maiores que 0,8 entre idades após $\mathrm{o}$ nascimento, e valores equivalentes à unidade

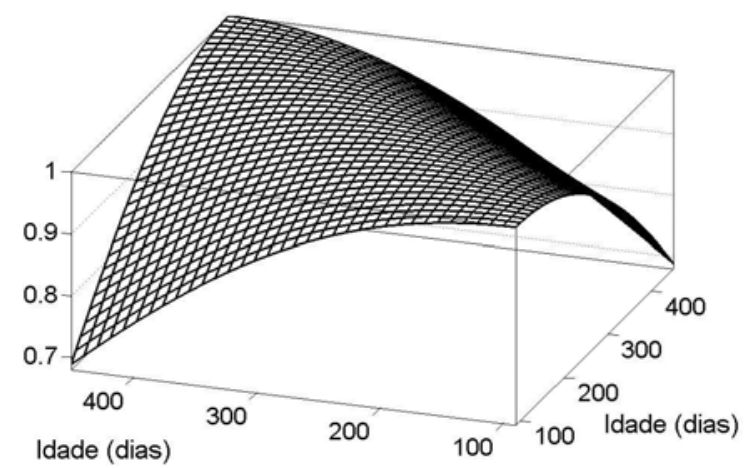

quando o intervalo entre idades era menor que três meses.

As correlações atribuídas aos efeitos genéticos aditivos maternos apresentaram-se em uma superfície com altos valores, à exceção das correlações entre as idades nos extremos do intervalo e as demais idades, que apresentaram depressão nos valores estimados. Essa deformação do padrão de correlação pode ser causada por artefatos do modelo ao estimar a estrutura de correlação nos extremos. Albuquerque e Meyer (2001) citaram como prováveis fontes de artefatos na estimativa da estrutura de correlação a pequena quantidade de dados nas idades extremas e a hiperparametrização do modelo. Porém, essas causas não podem ser utilizadas para explicar artefatos do modelo utilizado no presente estudo.

A estrutura de correlação estimada para o efeito permanente de ambiente apresentou valores próximos à unidade para qualquer combinação de pontos. Para um mesmo intervalo entre pontos, a correlação tornou-se maior quando esses pontos encontraram-se mais próximos do final do intervalo, o que pode ser explicado pelo caráter cumulativo desse efeito.

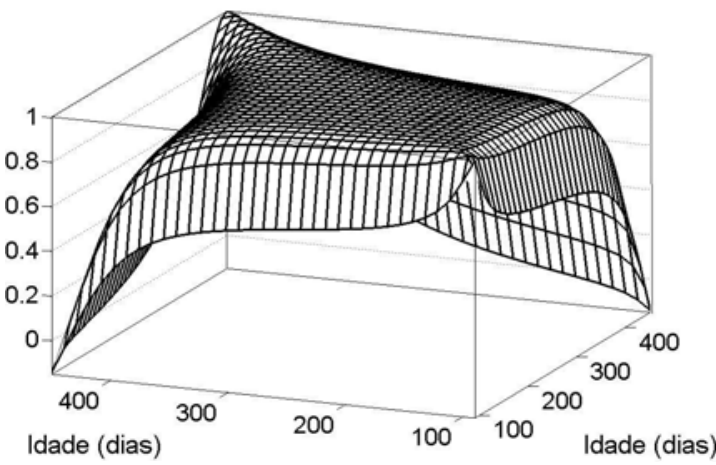

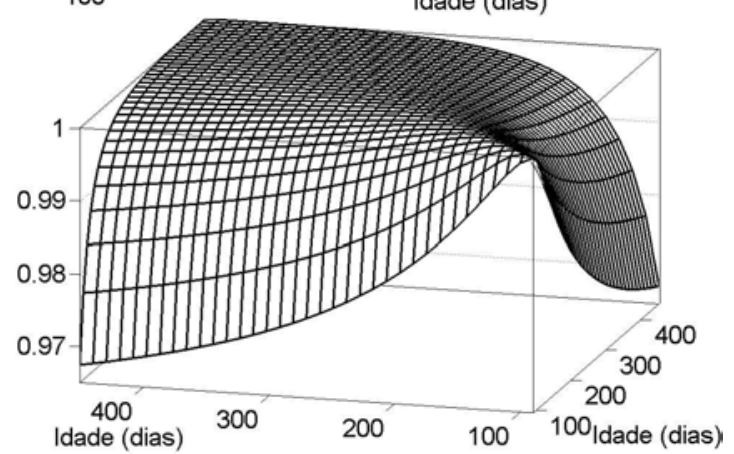

Figura 6. Correlações de peso em função da idade atribuídas aos efeitos genético aditivo direto (superior esquerdo), genético aditivo materno (superior direito) e permanente de ambiente (inferior) para dados referentes a ambas as regiões $(\mathrm{T})$. 


\section{CONCLUSÕES}

As trajetórias dos efeitos fixos em função da idade apresentam formas distintas (altura, inclinação e curvatura) para diferentes grupos contemporâneos e sugerem a necessidade de estimar um conjunto de coeficientes de regressão específico para cada grupo contemporâneo. A inexpressiva heterogeneidade de variância genética direta permite a comparação de valores genéticos aditivos diretos de animais avaliados em cada uma das duas regiões. Houve heterogeneidade de variância genética aditiva materna para peso entre 150 e 400 dias de idade nas duas regiões.

\section{REFERÊNCIAS BIBLIOGRÁFICAS}

ALBUQUERQUE, L.G. Regressão aleatória: nova tecnologia pode melhorar a qualidade das avaliações genéticas. In: SIMPÒSIO DA SOCIEDADE BRASILEIRA DE MELHORAMENTO ANIMAL, 5., 2004, Pirassununga. Anais... Pirassununga: SBMA, 2004.

ALBUQUERQUE, L.G.; MEYER, K. Estimates of covariance functions for growth from birth to 630 days of age in Nelore cattle. J. Anim. Sci., v.79, p.2776-2789, 2001

ARANGO, J.A.; CUNDIFF, L.V.; VAN VLECK, L.D. Covariance functions and random regression models for cow weight in beef cattle. J. Anim. Sci., v.82, p.54-67, 2004.

BERTRAND, J.K.; I. MISZTAL, I.; ROBBINS, K.R. et al. Implementation of random regression models for large scale evaluations for growth in beef cattle. In: WORLD CONGRESS ON GENETICS APLIED TO LIVESTOCK PRODUCTION, 8., 2006, Belo Horizonte. Proceedings... Belo Horizonte, 2006.

CUE, R.I.; WADE, K.M.; PIETERSMA, D. et al Genetic variability of growth curves in Quebec dairy heifers. In: WORLD CONGRESS ON GENETICS APLIED TO LIVESTOCK PRODUCTION, 8., 2006, Belo Horizonte. Proceedings... Belo Horizonte, 2006.

ELER, J.P.; VAN VLECK, L.D.; FERRAZ, J.B.S. et al. Estimation of variances due to direct and maternal effects for growth traits of Nelore cattle. $J$. Anim. Sci., v.73, p.3253-3258, 1995.
FALCONER, D.S. (Ed). Introduction to quantitative genetics. Essex: Longman Scientific \& Technical, 1989. 438p.

GILMOUR, A.R. Statistical models for multidimensional (longitudinal/spatial) data. In: WORLD CONGRESS ON GENETICS APLIED TO LIVESTOCK PRODUCTION, 8., 2006, Belo Horizonte. Proceedings... Belo Horizonte, 2006.

KIRKPATRICK, M.; HECKMAN, N. A quantitative genetic model for growth, shape, reaction norms, and other infinite-dimensional characters. J. Math. Biol., v.27, p.429-450, 1989.

LEGARRA, A.; MISZTAL, I.; BERTRAND, J.K. Constructing covariance functions for random regression models for growth in Gelbvieh beef cattle. J. Anim. Sci., v.82, p.1564-1571, 2004.

MARQUES, L.F.A.; PEREIRA, J.C.C.; OLIVEIRA, H.N. et al. Análise de características de crescimento da raça Simental. Arq. Bras. Med. Vet. Zootec., v.52, p.527-533, 2000.

MEYER, K. Bias and sampling covariances of estimates of variance components due to maternal effects. Genet. Sel. Evol., v.24, p.487-509, 1992.

MISZTAL, I. REMLF90 Manual, 2001. Disponível em:

$<$ http://nce.ads.uga.edu/ ignacy/newprograms.html/ $>$ Acessado em: 11 set. 2006

NOBRE, P.R.C.; LOPES, P.S.; TORRES, R.A. et al. Analyses of growth curves of Nellore cattle by Bayesian method via Gibbs sampling. Arq. Bras. Med. Vet. Zootec., v.55, p.480-490, 2003 a.

NOBRE, P.R.C.; MISZTAL, I.; TSURUTA, S. et al. Analyses of growth curves of Nellore cattle by multiple-trait and random regression models. $J$. Anim. Sci., v.81, p.918-926, 2003b

ROBBINS, K.R.; MISZTAL, I.; BERTRAND, J.K. A practical longitudinal model for evaluating growth in Gelbvieh cattle. J. Anim. Sci., v.83, p.2933, 2005.

SAKAGUTI, E.S. Funções de covariância $e$ modelos de regressão aleatória na avaliação genética de crescimento de bovinos jovens da raça Tabapuã. 2000. 81f. Tese (Doutorado) Universidade Federal de Viçosa, Viçosa, MG.

SCHAEFFER, L. R. 2001. Random regression. Disponível em: $<$ http://www.aps.uoguelph.ca/ lrs/ANSC637/LRS1 4/> Acessado em: 12 jan. 2001. 